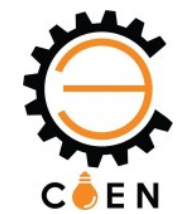

\title{
RADOMES COMPOSTOS POR ANÉIS RESSONADORES EM ANTENAS DE MICROFITA EM 18 GHZ
}

\author{
Marcelo A. Canavez ${ }^{(1)}$ (marcelocanavez2@gmail.com), Gláucio L. Ramos ${ }^{(2)}$ (glopesr@gmail.com), \\ Moacir J. Souza ${ }^{(3)}$ (moacir@ufsj.edu.br), Karina P. C. Souza ${ }^{(4)}$ (karinapirescs@gmail.com)

\footnotetext{
(1) Programa de Pós-Graduação em Engenharia Elétrica (UFSJ), São João del-Rei - MG, Brasil

(2) GAPEA - Grupo de Antenas, Propagação e Eletromagnetismo Aplicado (UFSJ), Ouro Branco - MG, Brasil

(3) Universidade Federal de São João del-Rei (UFSJ) - DETEM - Rodovia MG-443 - MG, Brasil

(4) Universidade Federal de São João del-Rei (UFSJ) - Rodovia MG-443 - MG, Brasil
}

RESUMO: Este artigo apresenta uma aplicação de estruturas periódicas de anéis repartidos ressonadores (SRR) quadrados em radomes para aumento de ganho e largura de banda em Antenas de Microfita (MPA) Retangulares. Foram feitas simulações através do software CST Microwave Studio ${ }^{\circledR}$, sendo apresentados o projeto da antena e do SRR para operação em $18 \mathrm{GHz}$. Foram utilizadas 49 células em cada camada de radome, em estruturas formadas por uma, duas ou três camadas. Foram feitas otimizações na célula via CST, buscando-se a máxima transmissão e mínima reflexão, sendo apresentado o gráfico para célula unitária. As distâncias entre as estruturas foram otimizadas buscando-se melhorias nas características da antena. São apresentados os gráficos da largura de banda, diretividade e ganho realizado da antena e dos conjuntos formados por uma antena com radomes de uma, duas e três camadas para frequências entre $16 \mathrm{GHz}$ e 20 $\mathrm{GHz}$. É apresentado ainda o diagrama de radiação tridimensional do ganho realizado da antena e do conjunto formado por antena e radome de três camadas. A estrutura proposta apresentou um aumento de ganho e diretividade nos três casos em relação à estrutura inicial, chegando a um aumento de 3,9 dBi em ganho realizado e de 33,33\% de largura de banda com a adição do radome de três camadas. Esta se mostrou uma alternativa viável ao ser comparada com conjuntos de antenas e com outras referências que utilizam estruturas feitas com ressonadores para melhoria das características das antenas de microfita.

PALAVRAS-CHAVE: Anéis Ressonadores Quadrados Repartidos, SRR, Antena de Microfita Retangular,

Radomes de elementos periódicos, Aumento de Ganho, Aumento de Largura de Banda.

\section{INTRODUÇÃO}

Antenas Patch Microfita (MPA) são largamente utilizadas em sistemas de comunicação sem fio e rádio. Dentre suas principais vantagens, podem-se citar sua pequena dimensão, fácil fabricação, baixo peso e baixo custo. Entretanto, apresentam duas desvantagens que limitam seu uso, a pequena largura de banda e ganho (Pozar, 2011; Balanis, 2016).

As estruturas periódicas de anéis ressonadores têm se mostrado promissoras na amenização destas desvantagens, tornando a MPA mais viável. Por isso, neste trabalho são usadas na forma de SRR quadradas, formando radomes que são posicionados acima da antena e melhoram suas características. Aplicações de estruturas ressonadoras em MPAs podem ser encontradas em Attia et. al (2009), Latrach et. al (2010), Xiaoqun (2016), Arora et. al (2017), Gangwar et. al (2017) e Patel et. al (2017).

As estruturas periódicas fabricadas artificialmente, também conhecidas por metamateriais, quando projetadas em conjunto e de forma geometricamente periódica, podem se comportar 

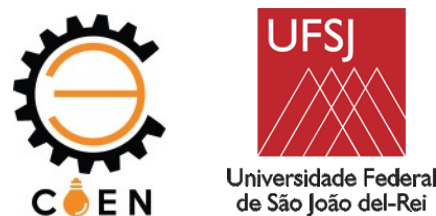

macroscopicamente como um único meio. Estas estruturas geralmente são compostas por anéis de material condutor que interagem com o campo magnético e trilhas estreitas que interagem com o campo elétrico, mas podem assumir outras formas. Estas estruturas, dependendo da forma que são dispostas, podem fornecer valores positivos ou negativos de permissividade, permeabilidade ou ambos (Veselago et. al, 2006; Smith et. al, 2000).

Estes anéis também possuem um gap em suas extremidades e serão melhor visualizados na Seção Referencial Teórico. Os efeitos dos gaps nas extremidades, do gap que separa os anéis e da espessura dos anéis foram investigados por Aydin et. al (2005). Aydin conclui que aumentar o gap das extremidades, entre os anéis e a espessura do metal, desloca eleva a frequência de ressonância. Adicionalmente, esta frequência é reduzida no caso de se inserir elementos capacitivos na estrutura.

\section{OBJETIVOS}

Neste trabalho o radome da antena é feito com células SRR, cada uma composta por dois anéis de cobre concêntricos, neste caso quadrados, sendo alinhadas periodicamente. Assim, pode ser aplicado o princípio de que ondas propagando em um meio composto por metamateriais muito menores do que o comprimento de onda, neste caso $\lambda / 10$, tem suas características próximas às dos elementos constituintes (Smith et. al, 2000). Este conceito é aplicado obtendo-se de coeficiente de reflexão e transmissão da célula, sendo considerado aproximado em relação ao meio.

Desta forma, buscou-se através da maximização das curvas de transmissão (S21) e minimização da reflexão (S11) na frequência desejada, obter melhorias do ganho e largura de banda da antena, pela otimização dos parâmetros da célula. São estudados três casos com um a três radomes colocados acima da antena para operação em $18 \mathrm{GHz}$, sendo comparados com Latrach et. al (2010), Xiaoqun (2016), Gangwar et. al (2017) e Souza (2018).

A busca pela otimização das curvas de transmissão e reflexão é similar ao que é feito por Gangwar et. al (2017). Contudo, este faz o uso de radome com quatro camadas formados por anéis ressonadores circulares para operação em $5.9 \mathrm{GHz}$, além de buscar índice de refração próximo de zero.

Latrach et. al (2010) utiliza também células quadradas SRR, com três radomes para melhoria das características de uma antena de microfita retangular. Contudo, este diferencia-se pela técnica de otimização voltada para minimização da parte imaginária da permeabilidade e pela frequência de operação de $2,17 \mathrm{GHz}$.

Xiaoqun (2016), utiliza uma estrutura de formato "S" de metamateriais de permeabilidade e permissividade com valores negativos para melhorar ganho e largura de banda em antenas de microfita operando em $5.89 \mathrm{GHz}$.

Os resultados simulados também são comparados com Souza (2018), de forma a se obter um parecer sobre a viabilidade da estrutura de três radomes apresentada em relação a um conjunto de duas antenas retangulares de microfita e em relação a dois conjuntos de duas antenas retangulares de microfita, ambas operando em $18 \mathrm{GHz}$.

\section{MATERIAIS E MÉTODOS}

As simulações desenvolvidas no presente trabalho foram feitas no CST Microwave Studio Suit ${ }^{\circledR}$ através do Time Domain Solver. Estas são formadas basicamente por simulações da célula unitária, da antena e do conjunto antena com radomes. Foi utilizado um material bom desempenho 

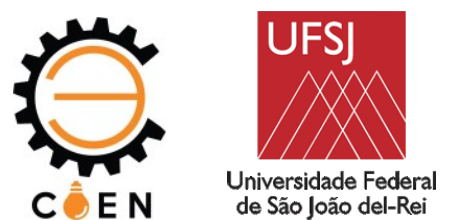

em altas frequências, o laminado Rogers ${ }^{\circledR}$ RO3003, com permissividade relativa $\left(\varepsilon_{r}\right)$ de $3,00 \pm 0,04$ e tangente de perdas 0,001 . Este apresenta espessura interior de $0,76 \mathrm{~mm}$ e na parte de cobre externa de $17,5 \mu \mathrm{m}$.

Para inserção da estrutura formada pelos radomes não foi necessária a modificação nas dimensões da antena. Isto permite que as características da antena projetada inicialmente sejam conservadas caso seja necessária a retirada de uma ou mais camadas radome para troca ou manutenção. A próxima Seção mostra as dimensões utilizadas no trabalho.

\section{REFERENCIAL TEÓRICO}

A célula metamaterial foi dimensionada inicialmente conforme Marwaha (2016) e em seguida otimizada para obter as características desejadas em $18 \mathrm{GHz}$. A otimização foi feita de forma a obter coeficientes de transmissão maximizados e reflexão minimizados.

A antena foi projetada para operar em $18 \mathrm{GHz}$. Uma vez que a trilha de alimentação de 50 ohms apresenta grande proporção em relação à largura da antena, tornou-se necessário a inserção de um casador de impedâncias, também conhecido como transformador de quarto de onda. Desta forma, a antena passa a ser alimentada por uma trilha mais estreita de $100 \mathrm{ohms}$ e o casador fica responsável por conectar a linha de $50 \mathrm{ohms} \mathrm{com} \mathrm{a} \mathrm{antena} \mathrm{projetada} \mathrm{para} 100 \mathrm{ohms}$. As equações utilizadas para projeto da antena e transformador de quarto de onda encontram-se em Pozar (2011), Balanis (2016). Pequenas modificações foram feitas na estrutura final para suavizar a transição entre o casador e a alimentação com $50 \mathrm{ohms}$ de impedância, inserindo-se uma pequena trilha de $0,5 \mathrm{~mm}$ de $50 \mathrm{ohms}$ antecedendo o mesmo. Buscou-se uma antena mais simétrica e consequentemente um radome também simétrico, apresentando melhorias nos resultados.

São apresentados nas Figuras 1,2 o projeto da célula metamaterial e da antena com transformador de quarto de onda, com dimensões em milímetros. A Figura 3 representa a parte superior do radome formado por 49 células, a Figura 4 representa o conjunto de antena e radome de três camadas e a Figura 5, os coeficientes de transmissão e reflexão.

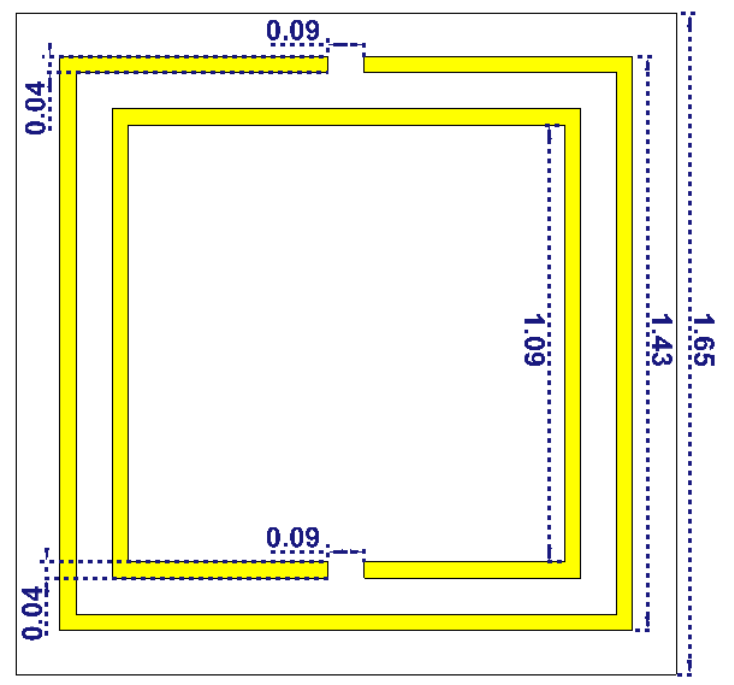

Figura 1. Estrutura da Célula unitária. 


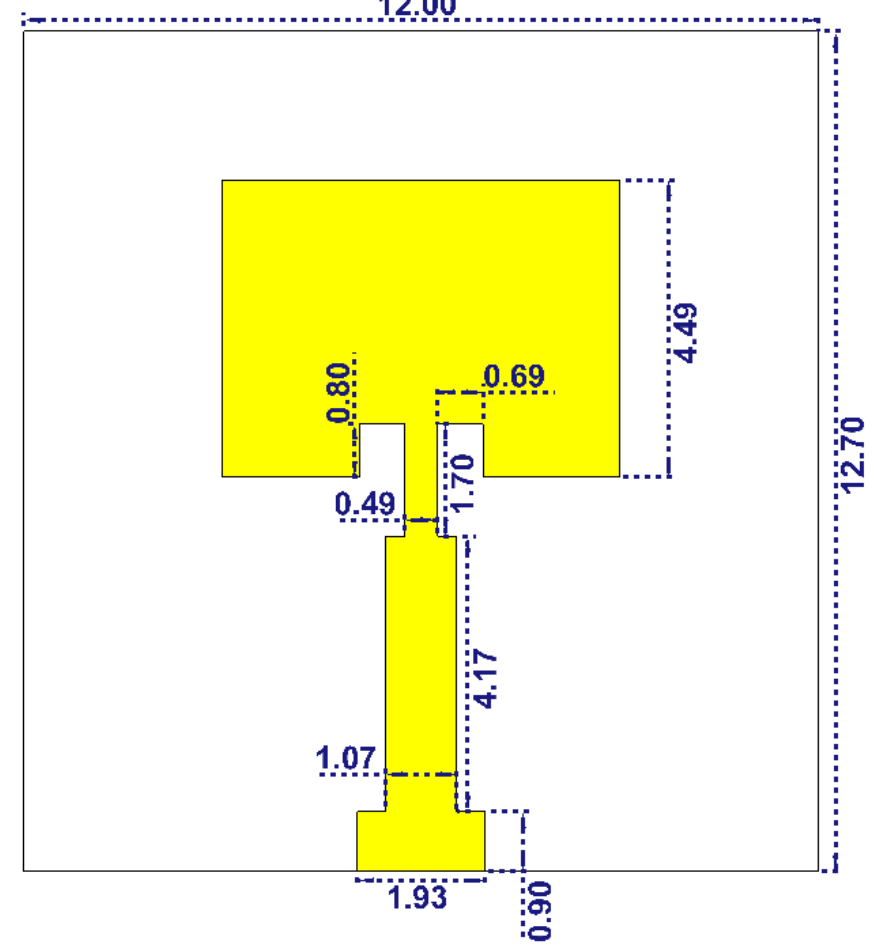

Figura 2. Antena Patch de Microfita com o casador de quarto de onda.

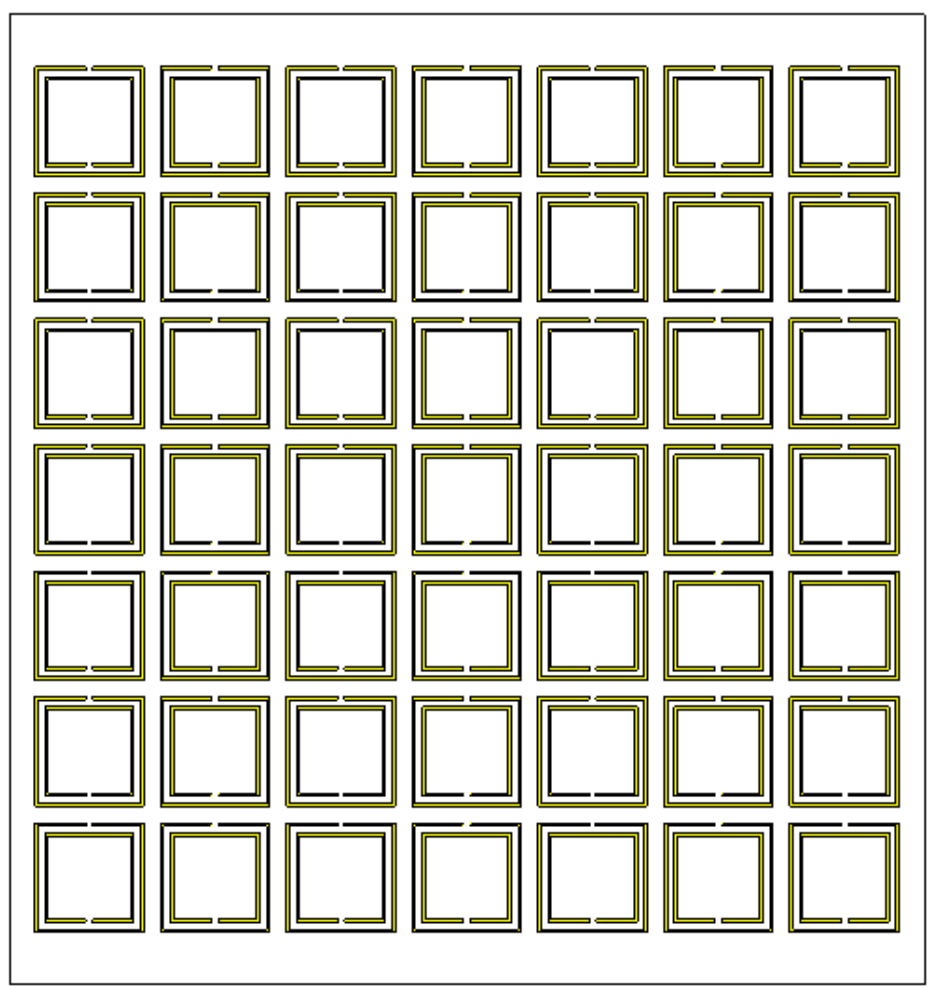

Figura 3. Recorte superior da estrutura geométrica do radome com 49 células. 

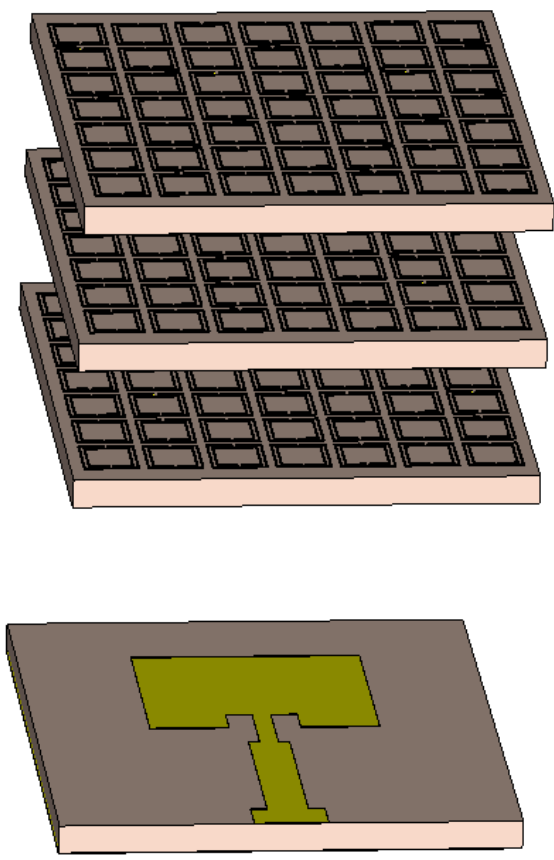

Figura 4. Estrutura completa composta por antena e radome de três camadas.

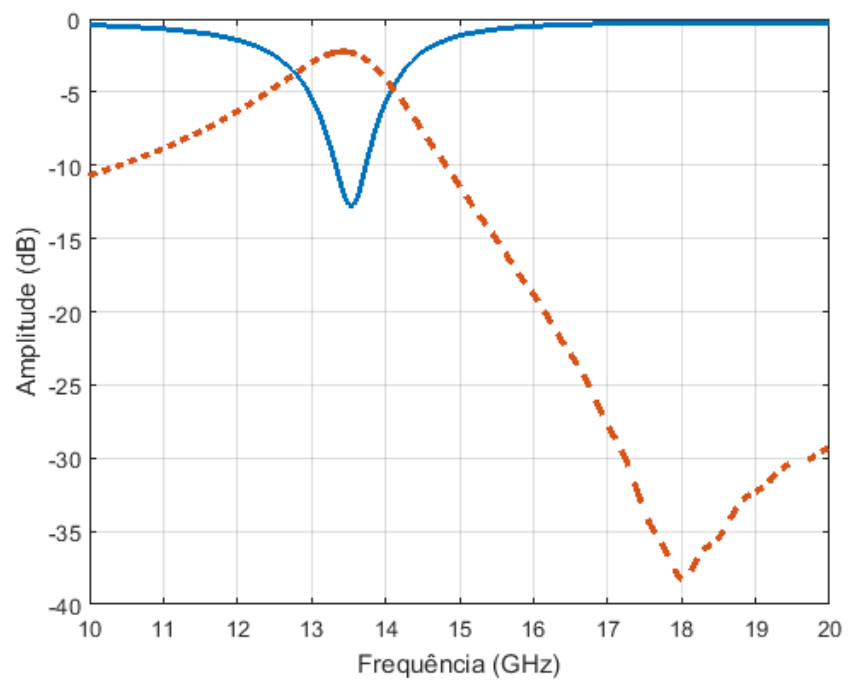

Figura 5. Coeficiente de transmissão (-) e coeficiente de reflexão (---) da célula metamaterial.

\section{RESULTADOS}

Esta Seção apresenta os resultados obtidos por simulação após inserção de uma, duas e três camadas de radomes. As distâncias foram otimizadas buscando-se o melhor ganho e largura de banda para frequência de $18 \mathrm{GHz}$. A variação da distância entre os radomes implica em curvas ganho versus frequência diferentes e em um deslocamento e atenuação na curva de coeficiente de reflexão, por isso, tornou-se necessário a otimização. As distâncias encontradas entre a antena e o conjunto de radomes e entre as camadas do radome variaram em cada caso e serão apresentadas na Seção Síntese. 
As curvas de coeficiente de reflexão, diretividade e ganho realizado em função da frequência para estas distâncias podem ser observadas nas Figuras 6, 7 e 8, respectivamente. Nestas Figuras, a cor verde em linha contínua representa a antena, as linhas tracejadas representam conjuntos com radomes, sendo a cor azul relativa ao radome de uma camada, rosa de duas camadas e preto de três camadas. As curvas relativas às Figuras 7 e 8 são relativas ao ângulo $\phi=90^{\circ}$. As Figuras 9 e 10 representam os diagramas tridimensionais de radiação em $18 \mathrm{GHz}$, sendo a primeira relativa à antena e a segunda ao conjunto de antena com radome de três camadas.

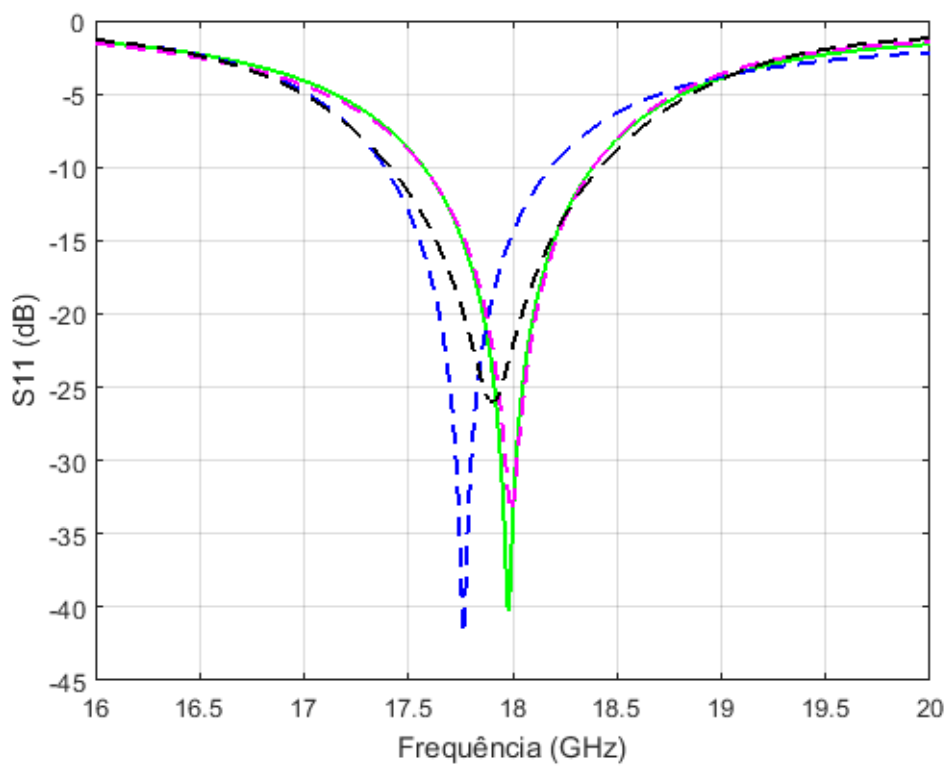

Figura 6. Coeficiente de reflexão da antena (-) e dos conjuntos com radomes (---). A cor verde representa a antena, azul o conjunto com radome de uma camada, rosa de duas e preto de três.

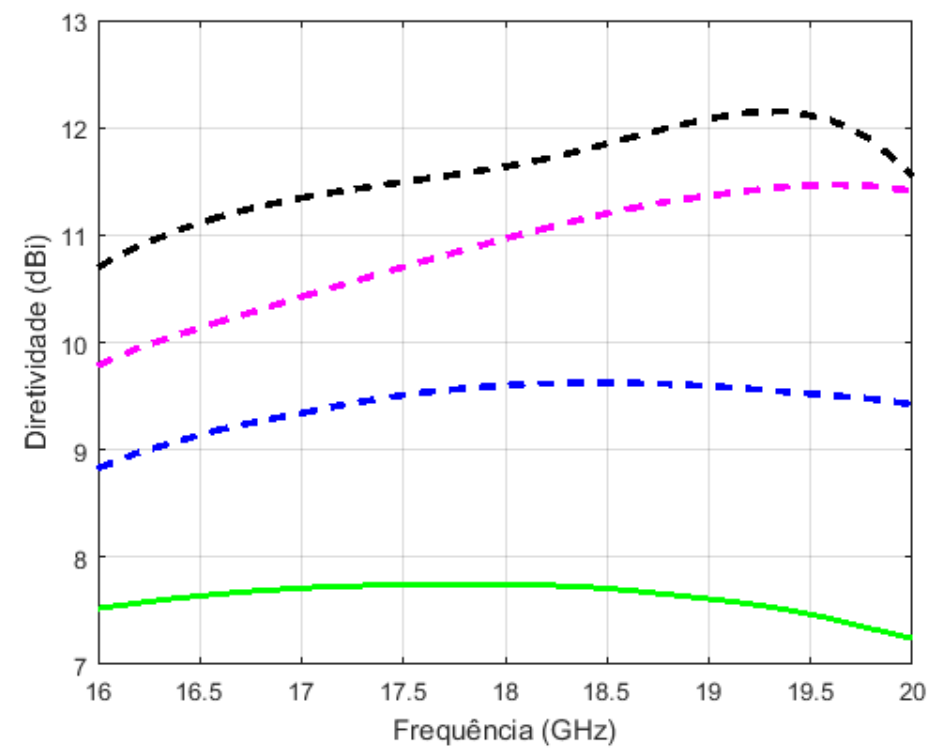

Figura 7. Diretividade da antena (-) e dos conjuntos com radomes (---). A cor verde representa a antena, azul o conjunto com radome de uma camada, rosa de duas e preto de três. 


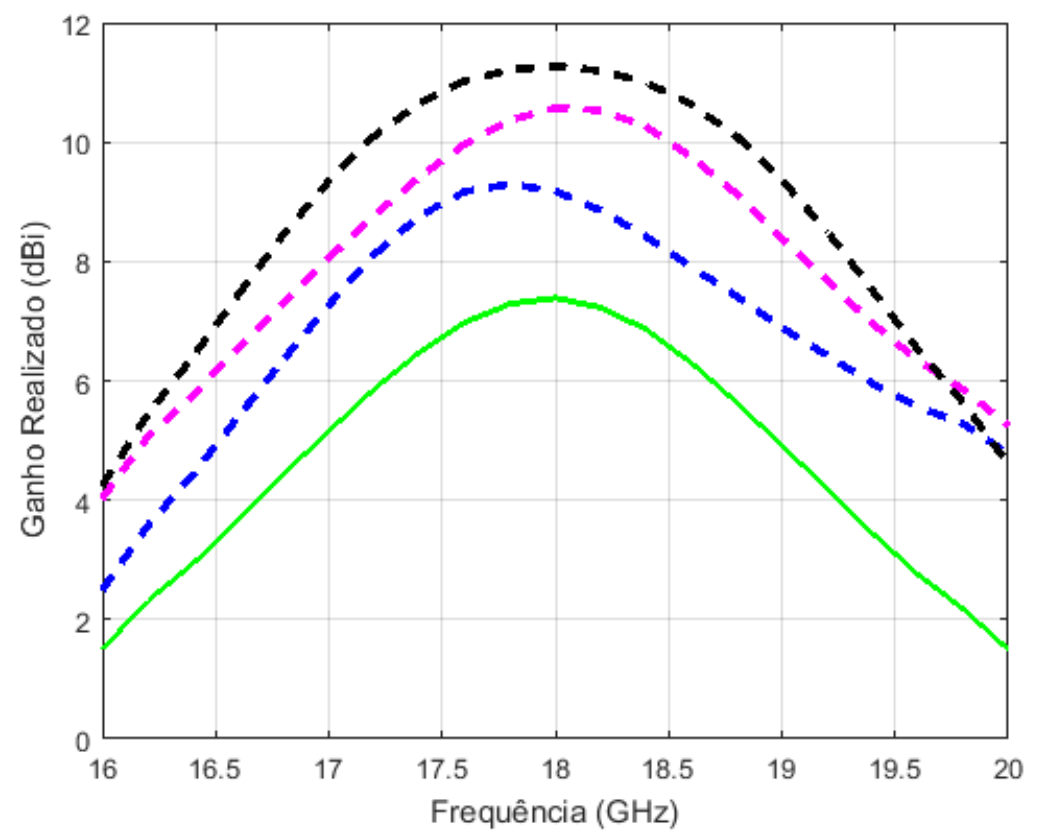

Figura 8. Ganho realizado da antena (-) e dos conjuntos com radomes (---). A cor verde representa a antena, azul o conjunto com radome de uma camada, rosa de duas e preto de três.

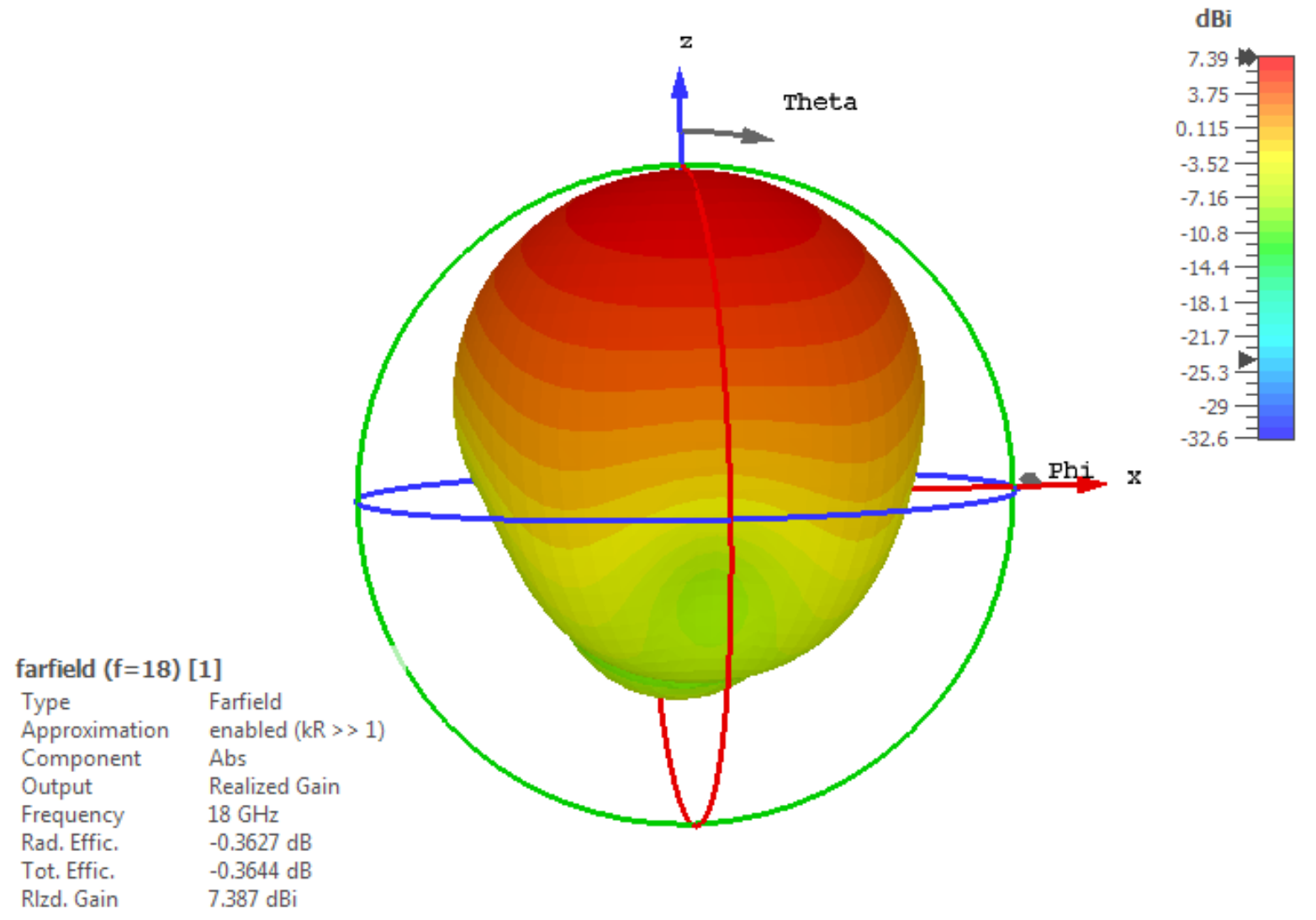

Figura 9. Diagrama de radiação da antena de microfita operando em $18 \mathrm{GHz}$. 

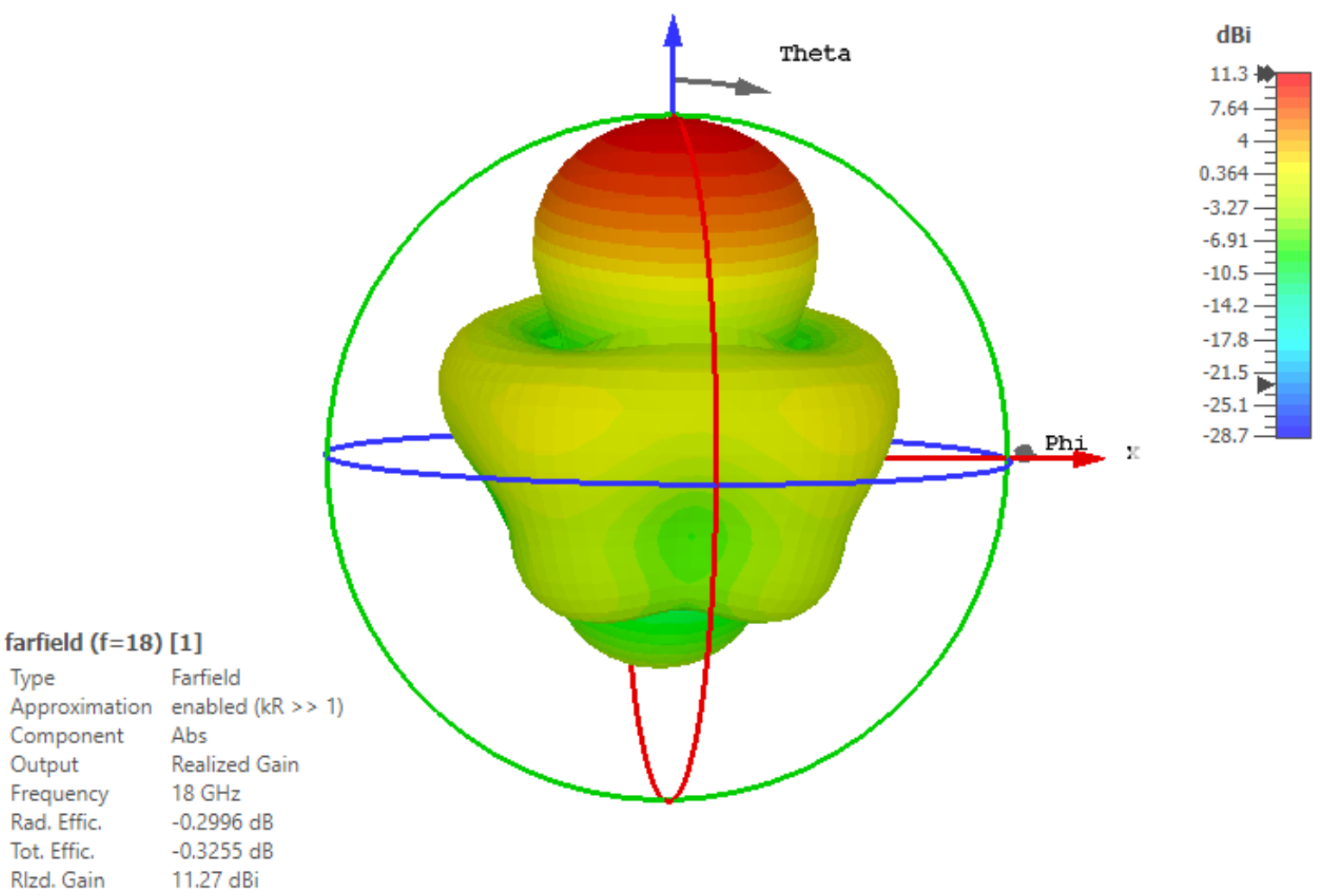

Figura 10. Diagrama de radiação da antena de microfita com três camadas de radome operando em

$18 \mathrm{GHz}$.

Pode ser observado, pela Figura 6, um deslocamento da frequência para o caso do radome de uma camada, uma curva bem próxima para o caso de duas camadas e um aumento na largura de banda para três camadas, sendo insuficiente para modificar a faixa de operação no segundo e terceiro casos. As Figuras 7 e 8 mostraram um aumento de ganho realizado e diretividade dentro da banda de operação para os três casos, partindo de $1,4 \mathrm{dBi}$ no pior caso, até aproximadamente $4 \mathrm{dBi}$ no melhor caso. Este aumento também pôde ser observado em menor escala fora da banda de operação. Os resultados para operação em $18 \mathrm{GHz}$ estão resumidos na Subseção Síntese.

Desta forma, se escolhido um valor limite de 7,4 dBi para ganho realizado, o mesmo da antena sem radome, como limite inferior aceitável permitido para o conjunto da antena com radome de três camadas, este teria um ganho aceitável no intervalo aproximado de $16,5 \mathrm{GHz}$ a $19,5 \mathrm{GHz}$. Assim, haveria um aumento de $270 \%$ na banda de operação, que passaria da operação de $0,81 \mathrm{GHz}$ para operação dentro de $3 \mathrm{GHz}$. Além disso, esta estrutura aumentou a diretividade, ganho realizado e largura de banda como visto nas Figuras 6 a 10.

\subsection{Síntese}

Nesta Subseção os resultados obtidos neste trabalho são sumarizados na Tabela 1. Em seguida, na Tabela 2, o melhor resultado é selecionado e comparado com os obtidos por Latrach et. al (2010), Gangwar et. al (2017), Souza (2018) e Xiaoqun (2016). Na Tabela 2, as estruturas estão representadas como: a antena do presente trabalho como Antena 1 e conjunto com radome de três 
camadas como Conjunto 1, a estrutura de Latrach et. al (2010) é representada por Antena 2 e Conjunto 2, de Gangwar et. al (2017) é representado por Antena 3 e Conjunto 3, Xiaoqun (2016) é representado por Antena 4 e Conjunto 4, Souza (2018) é representado por Antena 5 e Conjuntos 5 e 6. Os conjuntos escolhidos são melhor explicados na Seção Objetivos.

Tabela 1. Resumo dos resultados obtidos.

\begin{tabular}{|c|c|c|c|c|c|c|}
\hline Conjuntos & $\begin{array}{c}\text { Largura } \\
\text { de } \\
\text { Banda } \\
{[\mathrm{MHz}]}\end{array}$ & $\begin{array}{c}\text { Diretividade } \\
\text { [dBi] }\end{array}$ & $\begin{array}{c}\text { Ganho } \\
\text { realizado } \\
\text { [dBi] }\end{array}$ & $\begin{array}{c}\text { Distância } \\
\text { entre } \\
\text { antena e } \\
\text { primeira } \\
\text { camada } \\
\text { [mm] }\end{array}$ & $\begin{array}{l}\text { Distância } \\
\text { entre } \\
\text { primeira e } \\
\text { segunda } \\
\text { camada } \\
\text { [mm] }\end{array}$ & $\begin{array}{c}\text { Distância } \\
\text { entre } \\
\text { segunda e } \\
\text { terceira } \\
\text { camada } \\
\text { [mm] }\end{array}$ \\
\hline Antena & 810 & 7,77 & 7,39 & - & - & - \\
\hline $\begin{array}{c}\text { Conjunto } \\
\text { com } 1 \\
\text { radome }\end{array}$ & 798 & 9,60 & 9,18 & 9,1 & - & - \\
\hline $\begin{array}{l}\text { Conjunto } \\
\text { com } 2 \\
\text { radomes }\end{array}$ & 814 & 10,97 & 10,58 & 8,9 & 2 & - \\
\hline $\begin{array}{c}\text { Conjunto } \\
\text { com } 3 \\
\text { radomes }\end{array}$ & 1080 & 11,64 & 11,27 & 8,9 & 3 & 3 \\
\hline
\end{tabular}

Tabela 2. Comparação dos resultados.

\begin{tabular}{|c|c|c|c|c|}
\hline & $\begin{array}{c}\text { Largura de } \\
\text { Banda }[\mathrm{MHz}]\end{array}$ & $\begin{array}{c}\text { Diretividade } \\
\text { [dBi] }\end{array}$ & $\begin{array}{c}\text { Ganho } \\
\text { realizado } \\
{[\mathrm{dBi}]}\end{array}$ & Referência \\
\hline Antena 1 & 810 & 7,77 & 7,39 & Presente trabalho \\
\hline Antena 2 & - & 6,48 & 2,12 & Latrach et. al \\
\hline Antena 3 & 200 & - & 6,63 & Gangwar et. al \\
\hline Antena 4 & 1400 & - & 5,25 & Xiaoqun \\
\hline Antena 5 & 970 & - & 7,28 & Souza \\
\hline Conjunto 1 & 1080 & 11,64 & 11,27 & Presente trabalho \\
\hline Conjunto 2 & - & 9,38 & 5,57 & Latrach et. al \\
\hline Conjunto 3 & 200 & - & 14,23 & Gangwar et. al \\
\hline Conjunto 4 & 1640 & - & 9,19 & Xiaoqun \\
\hline Conjunto 5 & 840 & - & 9,61 & Souza \\
\hline Conjunto 6 & 1040 & - & 12,21 & Souza \\
\hline
\end{tabular}



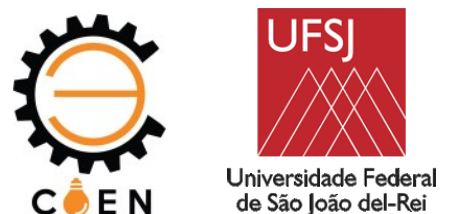

Os resultados obtidos mostraram uma melhora na largura de banda, diretividade e ganho realizado com a adição do radome com estruturas periódicas SRR. Contudo, a adição de cada radome melhora a performance da antena em uma proporção diferente, pois esta tem sua dinâmica modificada. Ao se adicionar a primeira camada do radome, analisando-se a frequência de operação de $18 \mathrm{GHz}$, aumentou-se aproximadamente 1,8 dBi de diretividade e ganho realizado, ao adicionar a segunda camada têm-se o aumento de aproximadamente $1,4 \mathrm{dBi}$ em diretividade e ganho realizado e, por fim, a adição da terceira camada tem-se a elevação de $0,69 \mathrm{dBi}$.

Os valores de ganho realizado obtidos para o caso do radome com três camadas, superam os obtidos por Latrach, Xiaoqun e do conjunto de duas antenas de Souza, sendo inferior ao conjunto de quatro antenas de Souza, e ao conjunto de Gangwar. Contudo, o conjunto proposto apresentou maior largura de banda que os outros conjuntos, com exceção de Xiaoqun.

Comparando-se mais especificamente com Souza, observou-se a viabilidade da estrutura apresentada em relação ao conjunto de duas antenas por apresentar um aumento de aproximadamente 1,6 dBi de ganho realizado e $240 \mathrm{MHz}$ de largura de banda. Contudo, este conjunto com três camadas se coloca entre o conjunto de duas antenas e quatro antenas, pois apresenta uma largura de banda ligeiramente menor e ganho realizado menor em aproximadamente $1 \mathrm{dBi}$, mas utilizando menos material proporcionalmente.

Os conjuntos de radomes de uma e duas camadas também se mostraram alternativas viáveis, pois além de não reduzir a largura de banda, elevaram o ganho realizado. Finalmente, a escolha da estrutura de SRR se mostrou uma alternativa para aumento de ganho realizado e no caso do radome de três camadas a largura de banda também. A escolha da estrutura a ser utilizada na prática, dependerá das exigências de ganho e largura de banda do projeto.

\section{CONCLUSÃO}

O objetivo de se melhorar o ganho e largura de banda da antena pôde ser alcançado com sucesso. A inserção das superfícies de radomes formados por células SRR confirmou as melhorias obtidas por outros trabalhos e se mostrou uma alternativa viável em relação ao conjunto de duas antenas de microfita para operação em $18 \mathrm{GHz}$, fornecendo ganhos superiores a 11,2 dBi e largura de banda de $1 \mathrm{GHz}$. O ganho realizado aumenta em relação à antena proposta inicialmente na medida em que são adicionadas camadas de radome, sendo este aumento de aproximadamente 1,79 dBi, 3,19 dBi e 3,88 dBi para uma, duas e três camadas, respectivamente. Pode ser observado também que o aumento é maior proporcionalmente com a adição da primeira camada em relação à adição da segunda e terceira camadas, possivelmente pela modificação na dinâmica do sistema. Contudo, este aumento em menor escala, principalmente no terceiro caso, é acompanhado pelo aumento da largura de banda. Esta apresenta um aumento de $270 \mathrm{MHz}$, ou seja, em 33,33\% em relação à configuração inicial e por isso torna atrativa a inserção do radome de três camadas.

\section{AGRADECIMENTOS}

Este trabalho foi parcialmente financiado por CAPES, CNPq e FAPEMIG. 

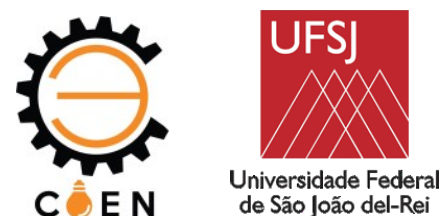

\section{DIREITOS AUTORAIS}

Os autores são os únicos responsáveis pelo conteúdo das informações contidas neste artigo.

\section{REFERÊNCIAS}

ARORA, Chirag; PATTNAIK, Shyam Sundar; BARAL, Rudra Narayan. SRR superstrate for gain and bandwidth enhancement of microstrip patch antenna array. Progress In Electromagnetics Research, 76, 73-85, 2017.

ATTIA, Hussein et al. Enhanced-gain microstrip antenna using engineered magnetic superstrates. IEEE Antennas and Wireless Propagation Letters, 8, 1198-1201, 2009.

AYDIN, Koray et al. Investigation of magnetic resonances for different split-ring resonator parameters and designs. New journal of physics, 7(1), 168, 2005.

BALANIS, Constantine A. Antenna theory: analysis and design. John wiley \& sons, 2016.

BANDEIRA K. M. C. Antena de Microfita com Metasuperfície para Sistemas Citizens Broadband Radio Service. XXXVI Simpósio Brasileiro de Telecomunicações e Processamento de Sinais SBrT2018, 2018.

GANGWAR, Deepak; DAS, Sushrut; YADAVA, R. L.. Gain enhancement of microstrip patch antenna loaded with split ring resonator based relative permeability near zero as superstrate. Wireless Personal Communications, v. 96, n. 2, p. 2389-2399, 2017.

LATRACH, M. et al. Design of a new type of metamaterial radome for low frequencies. Microwave and optical technology letters, v. 52, n. 5, p. 1119-1123, 2010.

LATRACH, M. et al. Design of a new type of metamaterial radome for low frequencies. 2010. [Online]. Disponível em: https://www.academia.edu/18329502/Design_of_a_new_type_of_ metamaterial_radome_for_low_frequencies. Acessado em 19 de maio, 2019.

MARWAHA, Anupma et al.. An Accurate Approach of Mathematical Modeling of SRR and SR for Metamaterials. Journal of Engineering Science \& Technology Review, v. 9, n. 6, 2016.

PATEL, Shobhit K.; ARGYROPOULOS, Christos; KOSTA, Yogeshwar P. Broadband compact microstrip patch antenna design loaded by multiple split ring resonator superstrate and substrate. Waves in Random and Complex Media, 27(1), 92-102, 2017.

POZAR, David M. Microwave Engineering. John Wiley \& Sons, 4th ed, 2011.

SMITH, D. R., Padilla, W. J., Vier, D. C., Nemat-Nasser, S. C., \& Schultz, S. Composite medium with simultaneously negative permeability and permittivity. Physical review letters, v. 84, n. 18, p. 4184, 2000.

SOUZA, K. P. C. Projeto e Simulação de Conjunto de Antenas de Microfita para Operação em 18 GHz. XVIII Simpósio Brasileiro de Microondas e Optoeletrônica - SBMC e XIII Congresso Brasileiro de Eletromagnetismo - CBMAG, 2018.

VESELAGO, Victor et al.. Negative refractive index materials. Journal of Computational and Theoretical Nanoscience, v. 3, n. 2, p. 189-218, 2006.

XIAOQUN, M. High Gain Microstrip Antenna with Left-Handed Materials. International Journal of Future Generation Communication and Networking, 9(3), 145-154, 2016. 


\title{
PERIODIC SPLIT RING RESONATOR BASED RADOME APPLIED TO MICROSTRIP ANTENNA AT 18 GHZ
}

\author{
Marcelo A. Canavez ${ }^{(1)}$ (marcelocanavez2@gmail.com), Gláucio L. Ramos ${ }^{(2)}$ (glopesr@gmail.com), \\ Moacir J. Souza ${ }^{(3)}$ (moacir@ufsj.edu.br), Karina P. C. Souza ${ }^{(4)}$ (karinapirescs@gmail.com)

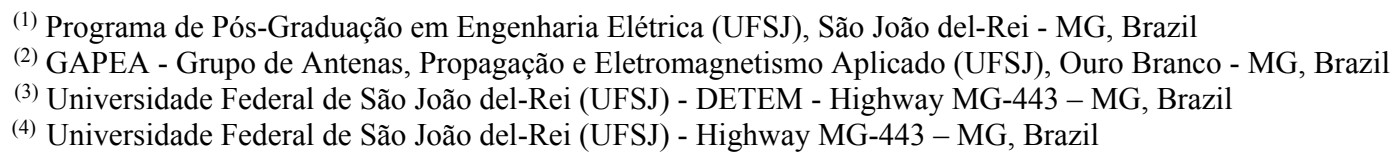

ABSTRACT: This paper presents an application of square Split Ring Resonators (SRR) aligned periodically forming radome structures in order to improve gain and bandwidth on rectangular Microstrip Antennas (MPA). Design simulations of the SRR and antenna at $18 \mathrm{GHz}$ were made using the CST Microwave Studio ${ }^{\circledR}$ software. Each radome layer were formed by either one, two or three layers of 49 cells. Optimizations were made in the cell dimensions via CST, aiming maximum transmission and minimum reflection and the resulting plot was presented. The distances between the structures were optimized aiming to improve antenna characteristics. Also, the plots of the return loss, directivity and realized gain of the antenna and of the arrays formed by the antenna with one, two and three layers radomes for frequencies of $16 \mathrm{GHz}$ to $20 \mathrm{GHz}$ were presented. Furthermore, the three-dimensional radiation diagram of realized gain of the antenna were presented. The diagram for the antenna with the three-layer radome were also presented. The proposed structure presented an increase in gain and directivity in all three cases in relation to the initial structure. As so, it reached a 3,9 dBi increase in realized gain and a 33,33\% increase in bandwidth with the insertion of the three-layer radome. This structure has proven to be a viable alternative when compared to other antenna sets and references that uses structures made of resonators to improve the characteristics of microstrip antennas.

KEYWORDS: Square Split Ring Resonators, SRR, rectangular Microstrip Antenna, periodic structure radome, Gain Improvement, Bandwidth Improvement. 